\title{
PRÁTICA DE ENFERMAGEM NA CONDIÇÃO CRÔNICA DECORRENTE DE HANSENÍASE ${ }^{1}$
}

\author{
Fabíola Rondon Freire da Silva², Aldenan Lima Ribeiro Corrêa da Costa ${ }^{3}$, Laura Filomena Santos de Araújo \\ Roseney Bellato ${ }^{5}$
}

\footnotetext{
${ }^{1}$ Estudo realizado como parte integrante do Sub-Projeto II da Pesquisa "Os desafios e perspectivas do SUS na atenção à saúde em municípios da área de abrangência da BR 163 no Estado de Mato Grosso" financiada pelo CNPq.

${ }^{2}$ Graduanda do curso de Enfermagem e Obstetrícia da Faculdade de Enfermagem (FAEN) da Universidade Federal do Mato Grosso (UFMT). Bolsista do CNPq. Mato Grosso, Brasil. E-mail: fabiolarondon@hotmail.com

${ }^{3}$ Doutora em Enfermagem. Professor Adjunto do Curso de Enfermagem da FAEN/UFMT. Mato Grosso, Brasil. E-mail: aldenan@yahoo.com.br

${ }^{4}$ Doutora em Enfermagem. Docente da FAEN/UFMT. Mato Grosso, Brasil. E-mail: laurafil1@yahoo.com.br

${ }^{5}$ Doutora em Enfermagem. Docente da FAEN/UFMT. Mato Grosso, Brasil. E-mail: roseney@terra.com.br
}

RESUMO: Objetivou-se compreender como se efetiva a prática de atendimento de enfermagem na prevenção da hanseníase e suas complicações, de modo a responder aos princípios da integralidade e resolutividade em duas unidades da Estratégia Saúde da Família na Regional de Saúde Diamantino-MT. Trata-se de pesquisa na modalidade estudo de caso, de natureza qualitativa, realizada no ano de 2007. A composição do corpus de análise compreendeu a observação direta das práticas de atendimento de enfermagem; produção de imagem das mesmas e análise documental. A análise dos dados evidenciou a relevância da prática de atendimento de enfermagem para os serviços de saúde. Também observou-se que essa prática é realizada de forma compartilhada com os demais membros da equipe de saúde, apontando para uma nova forma de atuar da enfermagem na qual há a possibilidade de ampliar a compreensão das necessidades de saúde das pessoas e propiciar a integralidade da atenção em saúde.

DESCRITORES: Enfermagem. Hanseníase. Assistência integral à saúde. Programa de saúde da família.

\section{NURSING CARE FOR CHRONIC HANSEN’S DISEASE}

\begin{abstract}
This study aimed at knowing how the nursing care in the prevention of Hansen's Disease as well as of its complications is carried out in order to meet the principles of integrality and resolutivity at two units from the Health Family Strategy in DiamantinoMT. This is a case study and it is a qualitative research, wich happened in the year of 2007. The analysis corpus consisted of direct observation of the nursing care practices; the production of images of these practices; and of analysis of documents. The data analysis revealed the importance of the nursing care practices to the health services. We have also observed that these practices are carried out together with the other members of the health staff indicating to nurses that there is a way of working wich brings the possibility of widening the understanding of people's health needs and of providing an integral health care.
\end{abstract}

DESCRIPTORS: Nursing. Leprosy. Comprehensive health care. Family health program.

\section{PRÁCTICA DE ENFERMERÍA EN LA CONDICIÓN CRONICA DE HANSENÍASE}

RESUMÉN: El objetivo de este estúdio fue comprender cómo se efectiva la práctica de la atención de enfermería en la prevención de la Hanseníase y sus complicaciones con el fin de responder a los principios de integridad y resolutividad en dos unidades del Estrategia de Salud de la Familia en Diamantino-MT en el año 2007. Se trata de una pesquisa en la modalidad estudio de caso, carácter cualitativo. La composición del cuerpo de análisis incluyó la observación directa de las prácticas de atención de enfermería; producción de imagen de la misma; análisis documental. El análisis de los datos mostró la pertinencia de la práctica de la atención de enfermería para los servicios de la salud. Observamos también que esta práctica es compartida con otros miembros del equipo de salud, apuntando a una forma de actuar de la enfermería en que hay posibilidad de ampliar la comprensión de las necesidades de salud, de las personas asistidas y proporcionar la integralidad de la atención de la salud.

DESCRIPTORES: Enfermería. Lepra. Atención integral de salud. Programa de salud familiar. 


\section{INTRODUÇÃO}

Este estudo foi realizado no âmbito da pesquisa "Os desafios e perspectivas do SUS na atenção à saúde em municípios da área de abrangência da BR 163 no Estado de Mato Grosso", coordenada pela Faculdade de Enfermagem (FAEN), e pelo Instituto de Saúde Coletiva da Universidade Federal de Mato Grosso (UFMT). Na sua realização foram selecionados quatro municípios da área de abrangência da BR 163: Diamantino, Alta Floresta, Sorriso e Sinop, os quais se constituem sede de Consórcios Intermunicipais de Saúde do Estado ou são cidades de referência para atenção em saúde. Também foram eleitas as seguintes práticas: $o$ atendimento de enfermagem; o planejamento estratégico; a regulação e a consulta médica; pressupondo que elas são realizadas mediante certa racionalidade que torna possível o atendimento à saúde das pessoas segundo o princípio da integralidade.

O foco deste estudo foi, dentre as práticas eleitas como domínio de análise, o atendimento de enfermagem a usuários da Regional de Saúde Diamantino (RSD) - MT vivenciando a condição crônica de hanseníase, pois o município é hiperendêmico para esse agravo de saúde. ${ }^{1}$

A hanseníase é uma doença infecto-contagiosa que representa um importante problema de saúde pública, não somente pelo grande número de pessoas que acomete, mas também pelas incapacidades que produz. Requer medidas que devem ser compartilhadas por todos os profissionais de saúde, em todos os níveis de atenção. O diagnóstico, tratamento e cura são possíveis primeiramente no âmbito da Atenção Básica (AB). As incapacidades físicas podem ser evitadas ou reduzidas, se as pessoas afetadas forem identificadas e diagnosticadas precocemente, tratadas com técnicas adequadas e acompanhadas nos serviços de saúde de AB. ${ }^{2}$

Em vista disso, práticas de enfermagem tornam-se de grande importância e estão particularmente associadas a prevenção das incapacidades e promoção da saúde, efetivadas principalmente por meio de educação em saúde no sentido de obter uma participação consciente e constante do usuário nos programas. Um grande desafio para a equipe de saúde é trabalhar na garantia de adesão dos usuários do Sistema Único de Saúde (SUS) ao tratamento, pois sabemos que a hanseníase exige um tratamento e acompanhamento de longo prazo. Conhecemos também as diversas reações causadas pelas medicações, dificultando esta adesão, razão pela qual se torna pertinente tomar as práticas de atendimento de enfermagem como objeto de estudo e análise.

Diante da predominância da hanseníase na população do município e da relevância da equipe de enfermagem para atuar na mudança dessa situação e no acompanhamento das pessoas que já vivenciam a experiência de sofrimento por esse agravo, questionamos neste estudo como acontece a Prática do Atendimento de Enfermagem (PAE) aos usuários com hanseníase na RSD.

As PAEs serão tomadas em nosso estudo como casos exemplares, através dos quais podemos indagar a lógica da integralidade segundo a qual os serviços de saúde operam. Tais práticas serão em nossa análise entendidas como formas particulares da experiência da integralidade da atenção em saúde, uma vez que se constituem como um momento privilegiado quando este princípio pode se materializar de forma singular e em certa medida.

O estudo objetivou compreender como se efetiva a PAE na prevenção da hanseníase e suas complicações, de modo a responder aos princípios de integralidade e resolutividade de usuários assistidos em unidades da Estratégia Saúde da Família (ESF) da RSD.

\section{METODOLOGIA}

Trata-se de pesquisa na modalidade estudo de caso, de natureza qualitativa, no sentido de que propicia aprofundar o conhecimento da realidade das práticas de enfermagem no SUS dentro da ESF no Estado de Mato Grosso.

Com base na experiência de adoecimento e busca por cuidados de um adolescente vivenciando complicações de hanseníase, foram selecionados os serviços de saúde por ele utilizados na RSD e, nesta regional, dois municípios. O município de Diamantino localiza-se na região dos Parecis, médio norte Mato-Grossense e possui uma população de 20.774 habitantes. É sede do Escritório Regional de Saúde, sendo referência em saúde para alguns municípios vizinhos, dentre os quais, São José do Rio Claro, com 14.690 habitantes. ${ }^{1}$

A coleta de dados foi realizada em duas unidades de ESF, nos municípios de Diamantino e São José do Rio Claro, no período de 27 a 30 de agosto de 2007, por uma equipe de seis pesquisadoras: uma doutora em enfermagem, uma mestranda vinculada ao Programa de Mestrado de Enfermagem e quatro alunas do Curso de Graduação em Enfermagem, da FAEN/UFMT. 
Nestes serviços, elegemos casos exemplares da prática do atendimento/consulta de enfermagem. Consideramos caso exemplar aquele que, em seu modo de apresentação, pudesse retratar as regularidades presentes na prática pesquisada, ou seja, seu modo de funcionamento e sua lógica.

Na apreensão da prática do atendimento/ consulta de enfermagem, interessou-nos sua materialidade discursiva e não discursiva. Com base no referencial teórico foucaultiano, materialidade discursiva são os discursos observados ou documentados, produzidos por profissionais; e materialidade não discursiva são as imagens e visibilidades dispostas no espaço e tempo, passíveis de descrições, tais como formas, processos, organizações, localizações, disposições, relações, procedimentos, técnicas e demais execuções. ${ }^{3}$ Assim, no trabalho de campo, aquilo que se encontrava na superfície e passível de ser apreendido com nosso olhar, ou com uma câmera fotográfica, foi tomado como manifestação da constituição das práticas profissionais investigadas.

Utilizamos, então, na composição do corpus de análise, três procedimentos distintos e complementares: observação direta das práticas - registradas no diário de campo; a análise de documentos oficiais do SUS; e a produção de "imagens exemplares" - registradas em instrumento específico contendo os elementos de identificação e autoria da imagem, bem como sua descrição de cenário e cena. Dessa forma foi eleito um conjunto de imagens que melhor pudesse nos ajudar a reconstituir "um modo de acontecer" da referida prática. Em sua leitura, este conjunto de imagens nos permitiu reconstituir a experiência, percorrer novamente os caminhos, seguir os sentidos que fomos dando no próprio ato de escolha de cada registro. Consideramos que este conjunto de procedimentos pôde apreender os elementos significativos das práticas investigadas.

O recurso da imagem oferece "um registro restrito, mas poderoso das ações temporais e dos acontecimentos reais". 4:137 São inúmeros os tipos de emprego de imagens com aplicações potenciais para pesquisa. Restringimo-nos a citar o trabalho do historiador social Aires, que empregou pinturas e gravuras para mostrar como na Europa préindustrial, concepções de crianças e da infância diferiam marcadamente daquelas das épocas mais recentes. Uma das razões essenciais da produção da imagem provém da vinculação desta com o domínio do simbólico, colocando-a em mediação entre o espectador e a realidade. ${ }^{5}$
$\mathrm{Na}$ análise, todo o material de campo foi descrito discursivamente, incluindo-se as imagens; seguido de sua leitura e análise numa perspectiva foucaultiana que considera o conteúdo e o contexto do discurso, mas não a sua estrutura, tal como é utilizado na linguística. Assim, os discursos se ligam a saberes, poderes e práticas, conforme Foucaulte teóricos pós-estruturalistas. ${ }^{6}$

Conforme está previsto na Resolução $\mathrm{N}^{\mathrm{O}}$ 196/96 do Conselho Nacional de Saúde, a qual regulamenta pesquisas que envolvem seres humanos, os cuidados éticos foram respeitados. Dessa forma, o projeto de pesquisa ao qual esse estudo está vinculado foi aprovado pelo Comitê de Ética e Pesquisa do Hospital Universitário Júlio Muller sob o No $235 / 05$.

\section{RESULTADOS E DISCUSSÃO}

Com base nas PAEs apreendidas, procedemos à análise temática, tendo como referenciais teóricos os princípios de integralidade e resolutividade em saúde e os estudos de Foucault.?

\section{Estratégias de atendimento: a busca por casos novos de hanseníase}

Durante a observação da PAE na regional de Diamantino, observamos várias estratégias adotadas para a identificação de pessoas afetadas por hanseníase, ou vulneráveis a tal condição. Além da visita domiciliar, realizada pelos Agentes Comunitários de Saúde (ACSs), da definição de um dia e horário específico para o atendimento a este problema, com consultas sistematicamente realizadas, outras formas de atendimentos são constituídas de acordo com as necessidades identificadas pela equipe de enfermagem, como descrito a seguir. [...] A enfermeira da ESF, juntamente com as ACSs, realizou um evento de educação em saúde denominado por elas "Mutirão da Hanseníase", caracterizado por palestras e teatros realizados em igrejas, fazendas e escolas do município, em um final de semana (Nota de Campo, 29/08/2007).

O Ministério da Saúde (MS) coloca como atribuição de toda a equipe da ESF a mobilização social em torno das demandas e necessidades em saúde, ações de promoção da saúde e ações educativas dirigidas à família e à comunidade. ${ }^{2} \mathrm{~A}$ busca e diagnóstico dos casos de hanseníase devem envolver práticas relevantes que contribuam para que o enfermeiro consiga estabelecer uma relação efetiva com os usuários. ${ }^{8}$ 
Ao analisarmos a atividade realizada, percebemos que a enfermeira e as ACSs fazem muito além do que é elaborado pelo MS, pois elas se utilizam da criatividade e produzem modos de atuar interessantes, que chamam a atenção da comunidade de maneira mais ampla e atrativa. Através da atividade desempenhada, a equipe de saúde integra as práticas de prevenção da doença, promoção da saúde e busca ativa por novos casos de hanseníase. Possibilitam, dessa maneira, o acesso da comunidade às informações, ao diagnóstico precoce, já interrompendo a cadeia de transmissão da doença. ${ }^{2}$ A integração de ações preventivas, promocionais e assistenciais implica a assimilação do princípio da integralidade em prol da reorientação de um modelo assistencial integral, humanizado e compromissado com o atendimento de necessidades e com a garantia do direito à saúde da população. ${ }^{9}$

A atitude do profissional que busca reconhecer as necessidades de ações de saúde relacionadas ao diagnóstico precoce, ou à redução de risco, ilustra um sentido da integralidade. Seja qual for a temática na qual o sentido da integralidade incide (políticas de saúde, organização dos serviços ou sobre as práticas), o que há em comum é a preocupação de discernir de um modo abrangente as necessidades dos usuários desses serviços, buscando gerar e aproveitar as oportunidades de aplicação das técnicas de prevenção, e, além disso, levar em conta as necessidades que não se reduzem à prevenção e ao controle das doenças. ${ }^{10}$ [...] a realização dessa atividade (Mutirão da Hanseníase) possibilitou o diagnóstico de 12 novos casos de hanseníase na população da área coberta pela ESF [...] (Nota de Campo, 29/08/2007).

Práticas como essas são essenciais, à medida que identificam pessoas com hanseníase precocemente, contribuem para a interrupção da cadeia de transmissão, resultando na diminuição dos índices de detecção da mesma, sendo esse um dos rumos certos para se alcançar o que é proposto pelo Plano de Eliminação da Hanseníase, ${ }^{11}$ ou seja, menos de um caso/10.000 habitantes até o ano de 2010.

Neste sentido, podemos afirmar que a PAE ao problema de hanseníase opera obedecendo as definições do MS, cuja racionalidade ainda é a anátomo-clínica. Mas ao colocar em movimento essa racionalidade, incorpora outras racionalidades, tais como a integralidade das ações de saúde, a integração dos serviços e o direito em saúde.

\section{A constituição da prática da consulta de enfermagem}

Compreendemos a Prática da Consulta de Enfermagem (PCE) não como algo natural, mas constituída ao longo da história no âmbito das relações sociais, logo, como um modo de atuar que opera uma dada racionalidade, participante dos jogos de poderes presentes nos discursos de verdades, aceitos em nossa sociedade.

"Cada sociedade tem seu regime de verda$\mathrm{de}^{\prime \prime}$, 7:12 ou seja, os tipos de discursos que são aceitos e que ditam a verdade das coisas e das práticas. Na sociedade ocidental nos guiamos por uma economia política da verdade, na qual se destacam as seguintes características: a verdade está centrada no discurso científico e suas instituições; produzimos discursos de verdade para favorecer a produção econômica e para dar sustentação ao poder político; os discursos de verdade precisam ser difundidos e consumidos e para isso precisam circular o mais amplamente possível; a produção e a circulação da verdade são realizadas sob certo controle, mas após ser produzida, a verdade precisa ser debatida por diferentes perspectivas ideológicas. Assim, para analisar os discursos de verdade, precisamos também considerar as redes nas quais os discursos estão envolvidos, seus diferentes campos de lutas e atravessamentos do poder. Logo, as práticas são produzidas pelos discursos, mas elas também os produzem. ${ }^{7}$

Tomando como referência os discursos de verdade descritos por Foucault, procuramos buscá-los na PAE, indagando dos discursos sobre saúde e enfermagem presentes em tal prática.

Apreendemos que o discurso que embasa o atendimento de enfermagem é o discurso científico anátomo-clínico com respaldo epidemiológico, mas tal discurso começa a ser permeado pelo discurso da integralidade da atenção em saúde, definido pelo SUS.

A consulta de enfermagem constitui-se e utiliza-se das fases propostas no processo de enfermagem, compreendendo cinco fases, sendo elas o histórico, exame físico, diagnóstico, prescrição e evolução de enfermagem. ${ }^{12}$ Tal prática é uma atividade independente, realizada pelo enfermeiro, objetivando propiciar condições para melhoria da qualidade de vida por meio de uma abordagem contextualizada e participativa.

No âmbito da ESF constatamos que a PCE tem grande relevância no serviço de saúde, pois 
em sua realização são consideradas as informações trazidas pelas pessoas que buscam a unidade, numa tentativa de considerá-las em suas múltiplas dimensões durante o atendimento. [...] Observamos a consulta de uma enfermeira a um usuário com suspeita de hanseníase. Ele havia procurado a ESF anteriormente e informado preocupação com uma mancha esbranquiçada e insensível na região supra maleolar lateral direita. Informou que havia queimado a mancha com cigarro em 5 áreas diferentes e, não sentindo dor, imaginou se tratar de hanseníase [...] (Nota de Campo, 28/08/2007).

A descoberta de novos casos de hanseníase também pode ocorrer através da detecção passiva, a qual acontece na própria unidade durante suas atividades gerais de atendimento à população. ${ }^{2}$ Entretanto, para que um usuário possa reconhecer os sinais da doença e tomar a iniciativa de procurar o serviço de saúde para esclarecer o diagnóstico, é necessário que esteja informado sobre esse agravo. Verificamos então, a importância da mobilização da comunidade, através de palestras educativas, material didático, dispositivos que proporcionem às pessoas conhecimentos sobre um problema de saúde.

Constatamos a interação entre a enfermeira e o médico para concluir o diagnóstico clínico da hanseníase, reforçando que o trabalho de enfermagem constitui uma prática social, quando o trabalhador interage diretamente com a pessoa por ele cuidada e com os demais trabalhadores de saúde. ${ }^{13}$ É essencial a interação dos profissionais de uma equipe, uma vez que isso reflete na qualidade do atendimento e na efetividade das ações, possibilitando uma maior resolutividade do problema de saúde apresentado pelo usuário. O diálogo entre diferentes sujeitos e seus distintos modos de percepção ampliam as possibilidades de apreensão das necessidades de saúde de uma pessoa ou um grupo populacional. ${ }^{9}$ A sequência da observação dessa prática também exemplifica um dos sentidos da integralidade: [...] A enfermeira afirma para o médico que ainda está preocupada com a percepção do usuário sobre a reabsorção do hálux, mas o médico argumenta que isso pode ser devido ao tipo de trabalho em lavoura realizado pelo usuário, como usa botina a maior parte do tempo, isso pode estar influenciando o posicionamento dos dedos [...] (Nota de Campo, 28/08/2007).

A preocupação da enfermeira está centrada na percepção do usuário acerca da perda de uma parte de seu corpo, independente de essa perda ser ou não consequência da hanseníase. Ainda que essa perda do hálux venha se dar em decorrência de outro motivo que não o atual agravo de saúde dessa pessoa, ela não deixará de ser uma perda, com uma repercussão importante em sua vida. A posição de cada um dos profissionais envolvidos nessa prática confirma as diferentes possibilidades de apreensão das necessidades de uma pessoa, de acordo com o olhar de cada sujeito. [...] Em seguida ela orienta o usuário sobre o uso das medicações que já haviam sido dadas ao mesmo no dia anterior, ou seja, mesmo o usuário não tendo sido examinado pelo médico ela já ofereceu a medicação e orientou o início do uso. Ela me esclarece que faz isso porque tinha certeza do diagnóstico médico e porque nesses casos de doença infecciosa a terapêutica deve ser iniciada o quanto antes, pois muitas vezes o usuário pode não retornar ou demorar a voltar e, enquanto isso poderá estar disseminando a doença [...] (Nota de Campo, 28/08/2007).

A legislação assegura ao enfermeiro como integrante da equipe de saúde, a "prescrição de medicamentos previamente estabelecidos em programas de saúde pública e em rotina aprovada pela instituição de saúde" ${ }^{14}$ sendo o caso da Poliquimioterapia (PQT) na hanseníase. Contudo, o discurso da enfermeira revela o controle exercido pelo discurso de verdade da medicina, ou seja, o poder-saber da clínica e do tratamento medicamentoso deve circular, podendo até mesmo ser exercido por outro profissional, mas o poder de diagnóstico é detido pelo médico. A enfermeira, por sua vez, exerce o seu poder de difundir o saber da clínica, mas incorpora outros saberes, como o saber de enfermagem sobre a terapêutica, a higiene e o conforto dos pés. [...] Ela mostra como é feito o controle da terapêutica de hanseníase e orienta o usuário a retornar para tomar no posto as medicações que precisam ser tomadas na presença dela [...] (Nota de Campo, 28/08/2007).

A enfermeira realiza as orientações ao usuário sobre como devem ser tomadas as medicações, buscando mantê-lo informado sobre como seguir seu tratamento, sendo esta uma maneira de promover a adesão ao mesmo, em longo prazo. Mais do que retornar ao posto para tomar as medicações sob a supervisão de um profissional, é enfatizado que a sua presença regular no serviço de saúde é importante para um acompanhamento contínuo da história de seu problema de saúde, de como ele está reagindo ao tratamento, das queixas, das alterações e das demais necessidades que ele possa apresentar.

A análise efetuada sobre a PCE evidencia um esforço por parte da enfermeira em efetivar o atendimento de acordo com a legislação sobre o controle 
da hanseníase no Brasil. ${ }^{15}$ Observa-se ainda o discurso anátomo-clínico como predominante, mas já evidenciando a introdução de um novo discurso, o da integralidade e da resolutividade da atenção em saúde, conforme definido nos princípios do SUS.

\section{O que um registro de atendimento pode trazer sobre a integralidade?}

Para discorrer sobre a análise da imagem exemplar da PAE, tivemos como referência que o registro fotográfico pode retratar uma prática, trazendo o modo como a mesma se configura em dado momento e lugar. Assim, o registro fotográfico da gaveta de prontuários nos mostra a imagem de uma gaveta de armário de aço que possui três gavetas, localizado atrás do balcão da recepção da unidade de ESF, junto a quatro armários com a mesma finalidade. Ele comporta as fichas de prontuário dos usuários da área de abrangência desse serviço, guardadas em pastas organizadas em ordem crescente pelo número das famílias. Para cada família há uma pasta mantida em gavetas separadas por microáreas. O prontuário é um documento de registro das informações do usuário, tais como problemas de saúde identificados, condutas adotadas, resultado de exames, procedimentos realizados na unidade, visitas domiciliares etc. Também é denominado como "ficha das famílias".

As informações são registradas por todos os integrantes da equipe multiprofissional da ESF, que realizam o atendimento a essas pessoas. Os registros são considerados produções e/ou documentos que contêm descrições de práticas profissionais e são entendidos como expressão dos modos de atuar dos sujeitos. ${ }^{3}$ Para isso, é necessário um registro claro de cada atendimento realizado, sendo esta uma maneira de documentar a prática efetuada.

O registro das ações da enfermagem é essencial para garantir a segurança, tanto do usuário como do profissional, possibilitando ainda a continuidade da assistência, quando da necessidade de atuação de outro profissional. ${ }^{16}$ É imprescindível que cada profissional identifique-se com os modelos a serem utilizados para registro, de forma clara, prática e objetiva.

Realizando a leitura de prontuários, foi possível compreender que os registros das PAEs realizados conforme o que cada profissional considera necessário sobre as práticas realizadas, ou sobre aquilo que ele consegue apreender, prever e responder.
[...]15.08.2007 - MH D - 12 doses $-1^{a}$ dose supervisionada[...](f21).

[...]20.04.2007 - Paciente com resultado de avaliação neurológica em hanseníase, realizado dia 19/04/2007. Paciente apresenta-se com o nervo radial $D$ e E espessado, nervo fibular direito e esquerdo, nervo tibial posterior D e E tínel. Paciente apresenta também perda/diminuição de sensibilidade do pé $D$ e E. Tendo grau de incapacidade I. Paciente fará tratamento para MH por 12 meses. Iniciando a $1^{a}$ dose na data de hoje e pedidos de exames de controle/avaliação. Informado sobre a importância de avaliar familiares. Relata que seu filho fez tratamento há 2 anos atrás. Realizar agendamento com familiares[...](f21).

Os dois registros de prontuários apresentados referem-se a atendimentos nos quais foram realizados diagnósticos de hanseníase multibacilar, efetuados por enfermeiros diferentes. No primeiro registro apenas consta que foi realizado o diagnóstico e administrada a primeira dose supervisionada do tratamento. Revela falta de informações sobre a história do usuário e sobre o atendimento realizado.

O segundo registro nos traz informações mais detalhadas no sentido de como acontece esse atendimento. Discorre sobre a leitura de um corpo sensorial da pessoa atendida, no que se refere às áreas exatamente afetadas pelo bacilo causador desse agravo. Para realizar essa leitura, o profissional que efetiva essa prática se utiliza de tecnologias de leitura, sendo tecnologia entendida "como um composto de saberes, procedimentos, $\mathrm{e}$ maquinarias, produzidos e operados pelos sujeitos das práticas" "3:81-2 No âmbito do nosso estudo, essas tecnologias podem ser entendidas como os saberes dos sujeitos operantes das práticas sobre hanseníase, as técnicas de avaliação funcional, bem como os instrumentos empregados nessa prática (estesiômetro, por exemplo).

Em seguida o profissional afirma o diagnóstico de hanseníase multibacilar e o tratamento que compreende 12 doses da PQT. Registra o relato do usuário sobre o fato de seu filho ter feito tratamento para hanseníase.

Estudos sobre os registros em prontuários afirmam que o relato das pessoas atendidas traz um potencial importante na produção de informações de grande relevância sobre os usuários. ${ }^{17}$ Além de o profissional exercitar o acolhimento, o olhar e a escuta, as informações recolhidas precisam também estar registradas, de modo a permitir a percepção da evolução do estado da pessoa atendida, do seu processo de adoecimento, das 
adaptações de vida e das respostas à sua condição de saúde e às propostas terapêuticas.

Estudiosos consideram que o enfermeiro ao estabelecer uma relação social com o assistido deve promover acolhimento em relação ao que é falado pelo usuário, de modo a facilitar a compreensão ampliada de sua história de vida. ${ }^{18}$

Outro momento significativo no atendimento refere-se às orientações que o usuário precisa receber acerca do seu problema de saúde, envolvendo especialmente seus familiares, visto estes terem maior contato com a pessoa com hanseníase.

Através dos registros da PAE às pessoas afetadas por hanseníase foi possível apreender que o discurso da integralidade, presente nas falas ainda não se traduz em prática concreta, pois se limita ao atendimento do corpo biológico. Os registros são sucintos e geralmente não abordam aspectos biopsicossociais das pessoas atendidas. Todavia, podemos ressaltar os esforços dos profissionais de enfermagem para dar corpo e visibilidade ao discurso da integralidade, quando observamos detalhes dessa prática, nos mutirões, palestras, teatros, realização de festividades como festas juninas e recreação infantil.

No decorrer dessas atividades e na descrição das mesmas pelos profissionais de enfermagem, pudemos evidenciar a consideração de outras dimensões das pessoas vivenciando a condição crônica de hanseniano, relacionadas principalmente à família, ao trabalho e ao lazer. Tais dimensões obrigam à superação do discurso anátomo-clínico e epidemiológico como verdade única a engendrar nossas práticas em saúde.

\section{CONSIDERAÇÕES FINAIS}

A atenção em enfermagem na prevenção da hanseníase e das complicações dela decorrentes é realizada em conformidade com o modelo bioclíni$\mathrm{co}$, sendo este também o modelo que orienta toda a equipe de saúde da ESF. Evidenciamos, contudo, a essencialidade da PAE para a efetivação dos princípios de integralidade e resolutividade a pessoas atendidas nas unidades da ESF da RSD que vivenciam a condição crônica por hanseníase.

Observamos que a PAE não se restringe ao consultório da unidade da ESF, nem ocorre individualmente pela enfermeira, mas é realizada de forma compartilhada com o médico e com os demais membros da equipe de enfermagem, apontando para uma nova forma de atuar da enfermagem, na qual há possibilidade de ampliar a compreensão das necessidades de saúde das pessoas atendidas e propiciar a integralidade da atenção em saúde. Ao realizar as ações propostas pelo MS expressas nos protocolos de hanseníase, a enfermeira interage com a equipe de saúde e busca modos interessantes de atuar, considerando a criatividade, a integralidade e o direito em saúde.

O estudo nos possibilitou questionar a PAE e sua relação com nossos discursos de produção de verdade na Enfermagem em Mato Grosso, revelando que a integralidade ainda se manifesta de forma tímida, mesmo sendo respaldada, como princípio constitucional. Permitiu também constatar que a visibilidade dessa integralidade exige uma maior disposição por parte dos profissionais de enfermagem a resistir ao biopoder no âmbito da ESF.

A efetivação da integralidade no cuidado em saúde será dependente dos resultados das disputas, sempre tensas, entre o discurso da Integralidade (favorável ao usuário do SUS) e o discurso da Biomedicina (favorável ao corporativismo médico). Tais discursos estão inscritos em jogos de poder-saber. ${ }^{7}$

Recomendamos a realização de novos estudos versando sobre a atenção em enfermagem a pessoas vivenciando a condição crônica de hanseniano, pois esta temática fala da constituição da enfermagem e das possibilidades da adoção de novas práticas em saúde. Assim, novos saberes se constituirão, permitindo encontrar outros caminhos para a consideração da integralidade em saúde, não apenas a pessoas vivenciando a condição crônica de hansenianos, mas a todas as pessoas com um problema de saúde.

\section{REFERÊNCIA}

1. Datasus do Brasil. Secretaria Executiva do Ministério da Saúde. Banco de Dados. [online]. [acesso em 2008 Abr. 12]. Disponível em: http:/ / w3.datasus.gov.br/ datasus/datasus.php

2. Ministério da Saúde (BR), Secretaria de Políticas de Saúde, Departamento de Atenção Básica. Guia para o Controle da hanseníase. Brasília (DF): MS, 2002.

3. Araújo LFS. Processos de subjetivação inscritos na constituição da experiência de si da/o enfermeira/o nas práticas assistenciais de um cenário de trabalho exemplar - a Unidade de Terapia Intensiva [tese online]. Ribeirão Preto (SP): Universidade de São Paulo. Programa de Pós-graduação em Enfermagem Fundamental; 2005. Disponível em http://www. teses.usp.br / teses / disponiveis / 22/22132/ tde-28112005-112621/

4. Loizos P. Vídeo, filme e fotografias como 
documentos de pesquisa. In: Martin W. Bauer, Geroge Gaskell, editores. Pesquisa qualitativa com texto: imagem e som: um manual prático. Petrópolis (RJ): Vozes; 2002.

5. Aumont J. A imagem. $10^{\text {a }}$ ed.Campinas (SP): Papirus; 1993.

6. Gore JM. Foucault e educação: fascinantes desafios. In: SILVA TT, organizador. O sujeito da educação: estudos foucaultianos. $5^{\mathrm{a}}$ ed. Petrópolis (RJ): Vozes; 2002. p.9-20.

7. Foucault M. Microfísica do poder. Organização, introdução e revisão técnica de Roberto Machado. $18^{\mathrm{a}}$ ed. Rio de Janeiro (RJ): Graal, 2003.

8. Pedrazzani ES. Levantamento sobre as ações de enfermagem no programa de controle da hanseníase no estado deSão Paulo. Rev Latino-am. Enfermagem. 1995 Jan; 3(1):109-15.

9. Alves VS. Um modelo de educação em saúde para o Programa Saúde da Família: pela integralidade da atenção e reorientação do modelo assistencial. Interface - Comunic., Saúde, Educ. 2005, Set-Fev, 9(16):39-52.

10. Mattos RA. Os Sentidos da Integralidade: algumas reflexões acerca de valores que merecem ser defendidos. In: Pinheiro R, Mattos RA, organizadores. Os sentidos da integralidade na atenção e no cuidado à saúde. Rio de Janeiro (RJ): UERJ, IMS/ ABRASCO; 2006.

11. Ministério da Saúde (BR), Programa Nacional de Eliminação da Hanseníase, Secretaria de Vigilância em Saúde, Departamento de Vigilância Epidemiológica. Plano Nacional de Eliminação da Hanseníase em nível municipal 2006-2010. Brasília (DF): MS, 2006.
12. Brasil. Resolução COFEN n ${ }^{\circ}$. 272/2002, de 27 de agosto de 2002. Dispõe sobre a Sistematização da Assistência de Enfermagem - SAE - nas instituições de saúde brasileira [online]. Rio de Janeiro: Conselho Federal de Enfermagem, 2002 [acesso em 2008 Jul 04]. Disponível em: http://www.portalcofen.com.br/2007/materias. asp?ArticleID $=7100 \&$ sectionID $=34$

13. Costa ALRC, Ramos FRS. O processo de construção do cuidado de si. In: Silva MA, organizadora. Diferentes olhares sobre o trabalho da enfermagem. Cuiabá (MT): EdUFMT, 2005.

14. Brasil. Decreto $N^{\circ} 94.406$, de 8 de junho de 1987. Regulamenta a Lei $\mathrm{N}^{\circ}$ 7.498, de 25 de junho de 1986 , que dispõe sobre o exercício da enfermagem, e dá outras providências. Brasília, 8 de junho de 1987.

15. Ministério da Saúde (BR), Secretaria de Políticas de Saúde, Departamento de Atenção Básica, Área Técnica de Dermatologia Sanitária. Legislação sobre o Controle da Hanseníase no Brasil. Brasília (DF): MS, 2000.

16. Bondan RMM. Consulta de enfermagem em saúde mental sob a perspectiva da teorista Hildegard Peplau [dissertação]. Rio Grande, (RS): Universidade Federal de Rio Grande. Programa de Pós-graduação em Enfermagem; 2006.

17. Bassan FB, Caetano R. Buscando um registro mais integral do cuidado à saúde: desenvolvimento, aplicação e avaliação de um novo instrumento de registro da consulta ambulatorial. Rev Bras Med. Fam Com. 2005 Out-Dez; 1(3):81-91.

18. Machado MTV, Leitão GCM, Holanda FUX. O conceito de ação comunicativa: uma contribuição para a consulta de enfermagem. Rev Latino-am Enfermagem. 2005 Set-Out; 13(5):723-8. 